\title{
ENERGY OF GLOBAL FRAMES
}

\author{
FABIANO G. B. BRITO and PABLO M. CHACÓN ${ }^{凶}$
}

(Received 22 April 2005; accepted 23 May 2005)

Communicated by C. D. Hodgson

\begin{abstract}
The energy of a unit vector field $X$ on a closed Riemannian manifold $M$ is defined as the energy of the section into $T^{1} M$ determined by $X$. For odd-dimensional spheres, the energy functional has an infimum for each dimension $2 k+1$ which is not attained by any non-singular vector field for $k>1$. For $k=1$, Hopf vector fields are the unique minima. In this paper we show that for any closed Riemannian manifold, the energy of a frame defined on the manifold, possibly except on a finite subset, admits a lower bound in terms of the total scalar curvature of the manifold. In particular, for odd-dimensional spheres this lower bound is attained by a family of frames defined on the sphere minus one point and consisting of vector fields parallel along geodesics.
\end{abstract}

2000 Mathematics subject classification: 53C20, 58C25.

Keywords and phrases: energy, vector fields, frames, parallel translation.

\section{Introduction}

Let $\left(M^{n+1}, g\right)$ be a compact oriented Riemannian manifold without boundary and Levi-Civita connection $\nabla$. Given a unit vector field $X$ on $M$, the energy of $X$ is (see [13])

$$
\mathcal{E}(X)=\frac{1}{2} \int_{M}\|\nabla X\|^{2}+\frac{n+1}{2} \operatorname{vol}(M) .
$$

Up to a constant, the first integral of (1) sometimes appears in the references as the total bending of $X$ or the vertical energy of the section of the tangent bundle.

The lower bound of the energy functional is $(n+1) \operatorname{vol}(M) / 2$, attained by parallel vector fields, that is, when $\nabla X=0$. For this kind of vector fields, the integral curves are geodesic and the orthogonal distribution $X^{\perp}$ is integrable and totally geodesic.

The first author is supported by CNPq (Brazil) Grant No. 301207/80 and by Fapesp (Brazil) Proj. Tem. No. 1999/02684-5. The second author is partially supported by MEC/FEDER Grant No. MTM2004-04934-C04-02 (Spain). This work has been carried out during a post-doctoral stay of the second author supported by DGU (Spain) No. HBE2002-008.

(C) 2008 Australian Mathematical Society 1446-7887/08 \$A2.00+0.00 
Parallel vector fields are rare because if $M$ admits a unit parallel vector field, then $M$ is locally a Riemannian product.

The first important result concerning the energy of vector fields appears over $\mathbf{S}^{2 k+1}$, where $k \geq 1$.

THEOREM 1.1 (Wiegmink [14]). Hopf vector fields on $\mathbf{S}^{3}$ are stable critical points of the energy.

Later on, using a very different approach, the following result was found.

THEOREM 1.2 (Brito [4]). Hopf vector fields are the only unit vector fields on $\mathrm{S}^{3}$ to minimize $\mathcal{E}$.

In dimension 5 and so on, Hopf vector fields are unstable critical points of $\mathcal{E}$, see [15]. The instability is obtained by parallel translation along the flow of a gradient field of the original Hopf vector field. At the limit of this sequence we have a vector field with one singularity and finite energy. This limit vector field was considered in [10] for another similar functional, the volume of a vector field, but is simply defined in the following way. Take $p \in \mathbf{S}^{n+1}$ and $v \in T_{p} \mathbf{S}^{n+1}$; if $x \in \mathbf{S}^{n+1} \backslash\{-p\}$, let $V_{\mathrm{P}}(x)$ be the vector obtained by parallel translation along the minimizing geodesic from $p$ to $x$. We say the vector field parallel along the geodesics to refer to this $V_{\mathrm{P}}$. For a more intuitive description of $V_{\mathrm{P}}$, see the final section.

In [15] it was conjectured that the energy of global fields had an infimum attained by $V_{\mathrm{P}}$ (with one singularity). As mentioned below, this conjecture was refuted in [3].

Following the literature, we find another special unit vector field on $\mathbf{S}^{n+1}$. Let $V_{\mathrm{R}}$, the radial vector field or North-South field, be the unit vector field tangent to the geodesics from a fixed point $p \in \mathbf{S}^{n+1}$. Note that $V_{\mathrm{R}}$ has two antipodal singularities, $\pm p$. This field plays a fundamental role in the study of the energy.

THEOREM 1.3 (Brito and Walczak [5]). The energy of any unit vector field $X$ with isolated singularities on $\boldsymbol{S}^{n+1}$, where $n \geq 3$, satisfies the inequality

$$
\mathcal{E}(X) \geq \frac{n^{2}+n-1}{2(n-1)} \operatorname{vol}\left(S^{n+1}\right),
$$

and equality holds if and only if $X=V_{\mathrm{R}}$.

At this point, the question of whether or not there is a global unit vector field minimum of the energy was still unanswered. This question was completely solved in [3] with the following result.

Theorem 1.4 (Borrelli, Brito and Gil-Medrano [3]). The infimum of $\mathcal{E}$ among all globally defined unit smooth vector fields on the spheres $\boldsymbol{S}^{2 k+1}$, where $k \geq 2$, is $\mathcal{E}\left(V_{\mathrm{R}}\right)$.

For more results on energy, see, for example, [2, 6, 8, 9] or [12].

In this paper we show why vector fields parallel along the geodesics (the $V_{\mathrm{P}}$ defined above) are important for the energy functional. With this, we provide a satisfactory answer to the motivation of the conjecture of [15]. 
Let us now consider a global unit frame $B=\left\{X_{1}, X_{2}, \ldots, X_{n+1}\right\}$ on $M$. We can define a new functional: the sum of the energies of all of the fields of the frame. That is, we set

$$
\mathcal{E}(B)=\sum_{i=1}^{n+1} \mathcal{E}\left(X_{i}\right)
$$

This functional also makes sense if the global frame is defined off a finite subset of $M$. In this case, the integral (1) of the energy of some $X_{i}$ can be divergent.

Here we prove the following theorem.

THEOREM 1.5. Let $M^{n+1}$, where $n \geq 3$, be a closed Riemannian manifold and $B=\left\{X_{1}, \ldots, X_{n+1}\right\}$ be an orthonormal frame on $M \backslash Z$ for a finite subset $Z \subset M$. Then,

$$
\mathcal{E}(B) \geq \frac{1}{n-1} \int_{M} \tau+\frac{(n+1)^{2}}{2} \operatorname{vol}(M),
$$

where $\tau$ is the scalar curvature of M. Moreover, equality holds if and only if each $X_{i}^{\perp}$ is integrable and the leaves are umbilic submanifolds.

Note that the right-hand side of the inequality does not depend on the frame. Thus, Theorem 1.5 provides a lower bound of the new functional. We apply this theorem to the spheres. Recall that $\mathbf{S}^{n+1}$ minus one point is parallelizable.

THEOREM 1.6. Given a finite subset $Z \subset S^{n+1}$, where $n \geq 3$, and $B$ a global orthonormal frame in $S^{n+1} \backslash Z$, then

$$
\mathcal{E}(B) \geq \frac{\left(n^{2}+2 n-1\right)(n+1)}{2(n-1)} \operatorname{vol}\left(S^{n+1}\right),
$$

and equality holds if and only if each vector field of $B$ has an orthogonal distribution integrable with umbilic leaves.

In $\mathbf{S}^{n+1} \backslash\{p\}$, a frame formed by fields $V_{\mathrm{P}}$ satisfies the condition to be a lower bound of $\mathcal{E}$.

COROLlaRY 1.7. The global frames on $S^{n+1} \backslash\{p\}$ obtained by parallel translation along the geodesics leaving - $p$ of a frame at the point - $p$ to attain the lower bound of Theorem 1.6. Moreover, in $\boldsymbol{S}^{n+1} \backslash\{p\}$ only this kind of frame attains the lower bound.

This property characterizes the vector fields parallel along the geodesics when the singular set $Z$ is just one point. That is, this kind of vector field gives the minimum if it is considered within a global frame. If we compare Theorems 1.3 and 1.6 , we observe that the minimum value of $\mathcal{E}(B)$ is not the sum of the infimum values of $\mathcal{E}(X)$. 


\section{Notation}

The energy of a smooth map $f:(M, g) \rightarrow(N, h)$ between Riemannian manifolds is defined by (see [7])

$$
\mathcal{E}(f)=\frac{1}{2} \int_{M}\|d f\|^{2} .
$$

A unit vector field $X$ on a closed Riemannian manifold $M^{n+1}$ is a section of the unit tangent bundle $\pi: T^{1} M \rightarrow M$. It is well known that $T^{1} M$ has a natural metric induced from $g$, usually called the Sasaki metric. For a precise definition, we refer to [1] or [11]. With this, from (2) we can obtain (1).

Since $X$ is globally defined, we have the subbundle of $T M$ determined by the vectors orthogonal to $X$ at each point of $M$. We denote this subbundle by $X^{\perp}$.

Let us take an orthonormal local frame $\left\{e_{1}, \ldots, e_{n}\right\}$ on $M$ adapted to $X$, that is, $e_{n+1}=X$. The second fundamental form $\mathcal{H}$ of $X^{\perp}$ is given by

$$
\mathcal{H}=\left(h_{i j}\right) \quad \text { where } h_{i j}=g\left(\nabla_{e_{i}} e_{j}, X\right), \quad i, j=1, \ldots, n .
$$

Recall that $\mathcal{H}$ will be symmetric if and only if $X^{\perp}$ is integrable. The second elementary symmetric function of $\mathcal{H}, \sigma_{2}(X)$, is given by

$$
\sigma_{2}(X)=\sum_{1 \leq i<j \leq n}\left(h_{i i} h_{j j}-h_{i j} h_{j i}\right)
$$

The Ricci curvature and $\sigma_{2}$ are related by the formula

$$
\int_{M} 2 \sigma_{2}(X)=\int_{M} \operatorname{Ricci}(X, X) .
$$

A proof of (3) for the compact case can be found in [4]. This equality also holds true by integrating over $M \backslash Z$, where $Z$ is a finite subset of $M$, see [5]. The scalar curvature $\tau$ of $M$ is given by

$$
\tau=\sum_{i=1}^{n+1} \operatorname{Ricci}\left(e_{i}, e_{i}\right)
$$

where $\left\{e_{i}\right\}_{i=1}^{n+1}$ is an orthonormal local frame.

Considering (1), a straightforward computation gives

$$
\|\nabla X\|^{2}=\sum_{i=1}^{n+1}\left\|\nabla_{e_{i}} X\right\|^{2}=\sum_{i, j=1}^{n} h_{i j}^{2}+\sum_{i=1}^{n} a_{i}^{2},
$$

where $a_{i}$ are the components of the acceleration of $X$, that is, $a_{i}=g\left(\nabla_{X} X, e_{i}\right)$ for an adapted orthonormal local frame. It is now clear that only unit parallel vector fields have energy equal to $((n+1) / 2) \operatorname{vol}(M)$. 


\section{Proof of Theorem 1.5}

Let us now prove Theorem 1.5. Given a global frame $B=\left\{X_{1}, \ldots, X_{n+1}\right\}$ on $M \backslash Z$, first of all we consider each vector field $X_{a}$, where $a=1, \ldots, n+1$, separately.

The integrand of the energy of $X_{a}(4)$ is

$$
\left\|\nabla X_{a}\right\|^{2}=\sum_{i, j \neq a}\left(h_{i j}^{a}\right)^{2}+\sum_{i \neq a} g\left(\nabla_{X_{a}} X_{a}, X_{i}\right)^{2}=\sum_{i, j \neq a}\left(h_{i j}^{a}\right)^{2}+\sum_{i \neq a}\left(h_{a a}^{i}\right)^{2} .
$$

From here, the sum of these factors for each $X_{a}$ is

$$
\begin{aligned}
\sum_{a=1}^{n+1}\left\|\nabla X_{a}\right\|^{2} & =\sum_{a=1}^{n+1}\left(\sum_{i, j \neq a}\left(h_{i j}^{a}\right)^{2}+\sum_{i \neq a}\left(h_{a a}^{i}\right)^{2}\right) \\
& =\sum_{a=1}^{n+1}\left(\sum_{i \neq a}\left(h_{i i}^{a}\right)^{2}+\sum_{\substack{i, j \neq a \\
i \neq j}}\left(h_{i j}^{a}\right)^{2}+\sum_{i \neq a}\left(h_{a a}^{i}\right)^{2}\right) \\
& =2 \sum_{a=1}^{n+1} \sum_{i \neq a}\left(h_{i i}^{a}\right)^{2}+\sum_{\substack{a=1 \\
i \neq j}}^{n+1} \sum_{\substack{i \neq j \\
i \neq j}}\left(h_{i j}^{a}\right)^{2} .
\end{aligned}
$$

Now, for a fixed $a$ we can rewrite these sums using the expressions

$$
\begin{aligned}
\sum_{\substack{i, j \neq a \\
i<j}}\left(h_{i i}^{a}-h_{j j}^{a}\right)^{2} & =\sum_{\substack{i, j \neq a \\
i<j}}\left(\left(h_{i i}^{a}\right)^{2}+\left(h_{j j}^{a}\right)^{2}\right)-2 \sum_{\substack{i, j \neq a \\
i<j}} h_{i i}^{a} h_{j j}^{a} \\
& =(n-1) \sum_{\substack{i \neq a \\
\text { i }}}\left(h_{i i}^{a}\right)^{2}-2 \sum_{\substack{i, j \neq a \\
i<j}} h_{i i}^{a} h_{j j}^{a},
\end{aligned}
$$

and

$$
\begin{aligned}
\sum_{\substack{i, j \neq a \\
i<j}}\left(h_{i j}^{a}+h_{j i}^{a}\right)^{2} & =\sum_{\substack{i, j \neq a \\
i<j}}\left(\left(h_{i j}^{a}\right)^{2}+\left(h_{j i}^{a}\right)^{2}\right)+2 \sum_{\substack{i, j \neq a \\
i<j}} h_{i j}^{a} h_{j i}^{a} \\
& =\sum_{\substack{i, j \neq a \\
i \neq j}}\left(h_{i j}^{a}\right)^{2}+2 \sum_{\substack{i, j \neq a \\
i<j}} h_{i j}^{a} h_{j i}^{a} .
\end{aligned}
$$


When $n \geq 3$, from (5), (6) and (7) we obtain

$$
\begin{aligned}
\sum_{a=1}^{n+1}\left\|\nabla X_{a}\right\|^{2} & \geq \frac{2}{n-1} \sum_{a=1}^{n+1}\left(\sum_{\substack{i, j \neq a \\
i<j}}\left(h_{i i}^{a}-h_{j j}^{a}\right)^{2}+2 \sum_{\substack{i, j \neq a \\
i<j}} h_{i i}^{a} h_{j j}^{a}+\sum_{\substack{i, j \neq a \\
i \neq j}}\left(h_{i j}^{a}\right)^{2}\right) \\
& \geq \frac{2}{n-1} \sum_{a=1}^{n+1}\left(2 \sum_{\substack{i, j \neq a \\
i<j}} h_{i i}^{a} h_{j j}^{a}+\sum_{\substack{i, j \neq a \\
i<j}}\left(h_{i j}^{a}+h_{j i}^{a}\right)^{2}-2 \sum_{\substack{i, j \neq a \\
i<j}} h_{i j}^{a} h_{j i}^{a}\right) \\
& \geq \frac{4}{n-1} \sum_{a=1}^{n+1} \sum_{\substack{i, j \neq a \\
i<j}}\left(h_{i i}^{a} h_{j j}^{a}-h_{i j}^{a} h_{j i}^{a}\right)=\frac{4}{n-1} \sum_{a=1}^{n+1} \sigma_{2}\left(X_{a}\right) .
\end{aligned}
$$

To complete the proof we integrate (8) over $M$ and use (3)

$$
\begin{aligned}
& \mathcal{E}(B)=\frac{1}{2} \sum_{a=1}^{n+1} \int_{M}\left\|\nabla X_{a}\right\|^{2}+\frac{(n+1)^{2}}{2} \operatorname{vol}(M) \\
& \geq \frac{2}{n-1} \int_{M} \sum_{a=1}^{n+1} \sigma_{2}\left(X_{a}\right)+\frac{(n+1)^{2}}{2} \operatorname{vol}(M) \\
& =\frac{1}{n-1} \int_{M} \sum_{a=1}^{n+1} \operatorname{Ricci}\left(X_{a}, X_{a}\right)+\frac{(n+1)^{2}}{2} \operatorname{vol}(M) \\
& =\frac{1}{n-1} \int_{M} \tau+\frac{(n+1)^{2}}{2} \operatorname{vol}(M) \text {. }
\end{aligned}
$$

In the first inequality of (8) we have disregarded $1-2 /(n-1)=(n-3) /(n-1)$ terms of type $\left(h_{i j}^{a}\right)^{2}$ with $i \neq j$. Also we have discarded the terms $\left(h_{i i}^{a}-h_{j j}^{a}\right)^{2}$ and $\left(h_{i j}^{a}+h_{j i}^{a}\right)^{2}$ with $i \neq j$. Thus, the inequality of the theorem will be an equality if and only if for all $a=1, \ldots, n+1$ we have

$$
h_{i i}^{a}=\lambda_{a} \quad \text { and } \quad h_{i j}^{a}=0 \quad \text { for } i \neq j .
$$

That is, the equality holds if and only if each distribution $X_{a}^{\perp}$ is integrable and the integral leaves are umbilic submanifolds.

\section{Consequences}

The proof of Theorem 1.6 follows directly from Theorem 1.5 using the fact that $\tau\left(\mathbf{S}^{n+1}\right)=n(n+1)$.

In the introduction we defined the vector fields parallel along the geodesics as in [10] or [15]. However, there is an alternative description that is more useful to prove the corollary. 
Choose in $\mathbb{R}^{n+1}$ a direction $v$ and consider the foliation $\mathcal{F}_{v}$ of $\mathbb{R}^{n+1}$ by straight lines with the chosen direction. Using the stereographic projection from the North pole $N \in \mathbf{S}^{n+1}$ over $\mathcal{F}_{v}$, we obtain a foliation of $\mathbf{S}^{n+1} \backslash\{N\}$ by circles. Let us define $V_{\mathrm{P}}$ as a unit tangent vector field to that foliation. The properties of the stereographic projection tell us that both definitions of $V_{\mathrm{P}}$ are equivalent.

It is now clear that the orthogonal distribution $V_{\mathrm{P}}^{\perp}$ is integrable. Note that $\mathcal{F}_{v}^{\perp}$ in $\mathbb{R}^{n+1}$ is the $n$-dimensional foliation determined by the hyperplanes orthogonal to $v$. Thus, the leaves of $V_{\mathrm{P}}^{\perp}$ are $n$-dimensional spheres of $\mathbf{S}^{n+1}$. Recall that $V_{\mathrm{P}}$ is defined on $\mathbf{S}^{n+1} \backslash\{N\}$. The integral curves of $V_{\mathrm{P}}$ and also the leaves of $V_{\mathrm{P}}^{\perp}$ all meet at $N$. Obviously, we can define a congruent vector field parallel along the geodesics on $\mathbf{S}^{n+1} \backslash\{p\}$ for any $p \in \mathbf{S}^{n+1}$.

The properties of symmetry of $V_{\mathrm{P}}$ allow us to construct an orthonormal global frame on $\mathbf{S}^{n+1} \backslash\{p\}$ formed by $n+1$ fields parallel along the geodesics. In this way, we obtain an orthonormal global frame $B$ such that each distribution orthogonal to each field of $B$ is integrable with umbilic leaves. Therefore, such a $B$ satisfies the conditions of Theorem 1.6 to minimize the functional energy of frames. This argument proves the first statement of the corollary. Conversely, totally umbilical foliations in $\mathbf{S}^{n+1} \backslash\{p\}$ goes to totally umbilical foliations in $\mathbb{R}^{n+1}$ through the stereographic projection. Necessarily this kind of foliation in $\mathbb{R}^{n+1}$ is given by parallel hyperplanes, so in $\mathbf{S}^{n+1}$ these foliations are congruent to some $V_{\mathrm{P}}^{\perp}$. With that, an orthonormal frame satisfying the equality condition in Theorem 1.6 must be formed by fields $V_{\mathrm{P}}$. This complete the proof of the corollary.

We can obtain the energy of $V_{\mathrm{P}}$ from Theorem 1.6 (see also [15]):

$$
\mathcal{E}\left(V_{\mathrm{P}}\right)=\frac{n^{2}+2 n-1}{2(n-1)} \operatorname{vol}\left(\mathbf{S}^{n+1}\right) .
$$

When $n \geq 3$, this value is between the energy of the radial vector field and that of the Hopf vector field, $V_{\mathrm{H}}$ :

$$
\mathcal{E}\left(V_{\mathrm{R}}\right)=\frac{n^{2}+n-1}{2(n-1)} \operatorname{vol}\left(\mathbf{S}^{n+1}\right) ; \quad \mathcal{E}\left(V_{\mathrm{H}}\right)=\frac{2 n+1}{2} \operatorname{vol}\left(\mathbf{S}^{n+1}\right) .
$$

Note that $V_{\mathrm{R}}$ and $V_{\mathrm{H}}$ have the same energy on $\mathbf{S}^{3}$. On $\mathbf{S}^{n+1}$, individually, the radial vector field has less energy than the vector field parallel along the geodesics, but it is not possible to generate an orthonormal frame formed just by radial vector fields.

Finally, observe that Theorem 1.5 can be applied to manifolds other than spheres; parallelizable manifolds (Lie groups and others) for example.

\section{References}

[1] D. E. Blair, Riemannian Geometry of Contact and Symplectic Manifolds, Progress in Mathematics, 203 (Birkhäuser, Boston, 2002).

[2] E. Boeckx and L. Vanhecke, 'Energy of radial vector fields on compact rank one symmetric spaces', Ann. Global Anal. Geom. 23 (2003), 29-52. 
[3] V. Borrelli, F. Brito and O. Gil-Medrano, 'The infimum of the energy of unit vector fields on odd-dimensional spheres', Ann. Global Anal. Geom. 23 (2003), 129-140.

[4] F. G. B. Brito, 'Total bending of flows with mean curvature correction', Differential Geom. Appl. 12 (2000), 157-163.

[5] F. G. B. Brito and P. Walczak, 'On the energy of unit vector fields with isolated singularities', Ann. Math. Polon. 73 (2000), 269-274.

[6] P. M. Chacón and A. M. Naveira, 'Corrected energy of distributions on Riemannian manifolds', Osaka J. Math. 41 (2004), 97-105.

[7] J. Eells and L. Lemaire, 'A report on harmonic maps', Bull. London Math. Soc. 10 (1978), 1-68.

[8] O. Gil-Medrano, 'Volume and energy of vector fields on spheres. A survey', in: Differential Geometry, Valencia, 2001 (World Scientific, Singapore, 2002), pp. 167-178.

[9] O. Gil-Medrano, J. C. González-Dávila and L. Vanhecke, 'Harmonicity and minimality of oriented distributions', Israel J. Math. 143 (2004), 253-279.

[10] S. Pedersen, 'Volume of vector fields on spheres', Trans. Amer. Math. Soc. 336 (1993), 69-78.

[11] T. Sakai, Riemannian Geometry, Translations of the Mathematical Monographs, 149 (American Mathematical Society, Providence, RI, 1996).

[12] M. Salvai, 'On the energy of sections of parallelizable sphere bundles', Rend. Sem. Mat. Univ. Politec. Torino 60 (2002), 147-155.

[13] G. Wiegmink, 'Total bending of vector fields on Riemannian manifolds', Math. Ann. 303 (1995), 325-344.

[14] _ _ 'Total bending of vector fields on the sphere $S^{3}$, Differential Geom. Appl. 6 (1996), 219236.

[15] C. M. Wood, 'The energy of Hopf vector fields', Manuscripta Math. 101 (2000), 71-88.

\author{
FABIANO G. B. BRITO, Departamento de Matemática, \\ Instituto de Matemática e Estatística, Universidade de São Paulo, \\ Rua do Matão 1010, São Paulo-SP 05508-090, Brazil \\ e-mail: fabiano@ime.usp.br
}

PABLO M. CHACÓN, Departamento de Matemáticas, Facultad de Ciencias, Universidad de Salamanca, Plaza de la Merced 1-4, 37008 Salamanca, Spain e-mail: pmchacon@usal.es 